DE

M E D I C I N A

T R O P I C A L

$\mathrm{DE}$

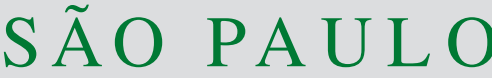

JOURNAL OF THE SÃO PAULO INSTITUTE OF TROPICAL MEDICINE

(1) Instituto Adolfo Lutz, Laboratório de Vírus Respiratórios, Núcleo de Doenças Respiratórias, CV/CCD/SES/SP, São Paulo, São Paulo, Brazil

(2) Centers for Disease Control and Prevention, Influenza Division, Atlanta, GA, USA.

Correspondence to: Terezinha Maria de Paiva.

Instituto Adolfo Lutz, Núcleo de Doenças Respiratórias, Av. Dr. Arnaldo, 355, CEP 01246-902, São Paulo, SP, Brasil. Tel: +55 11 3068-2913. Fax: +55 11 30853505.

E-mail: tterezinha@uol.com.br

Received: 15 July 2016

Accepted: 22 September 2016

\section{Molecular epidemiology of influenza A(H1N1)PDM09 hemagglutinin gene circulating in São Paulo State, Brazil: 2016 anticipated influenza season}

Katia Corrêa de Oliveira Santos ${ }^{1}$, Daniela Bernardes Borges da Silva ${ }^{1}$, Norio Augusto Sasaki ${ }^{1}$, Margarete Aparecida Benega1, Rebecca Garten ${ }^{2}$, Terezinha Maria de Paiva ${ }^{1}$

\section{ABSTRACT}

Compared to previous years, seasonal influenza activity commenced early in São Paulo State, Brazil, Southern hemisphere during the 2016 year. In order to investigate the genetic pattern of influenza $\mathrm{A}(\mathrm{H} 1 \mathrm{~N} 1)$ pdm09 in the State of Sao Paulo a total of 479 respiratory samples, collected in January by Sentinel Surveillance Units, were screened by real-time RT-PCR. A total of 6 Influenza viruses A(H1N1)pdm09 presenting ct values $\leq 30$ were sequenced following phylogenetic analysis. The present study identified the circulation of the new 6B.1 subgroup (A/Sao Paulo/10-118/2016 and A/Sao Paulo/3032/2016). In addition, influenza $\mathrm{A}(\mathrm{H} 1 \mathrm{N1})$ pdm09 group 6B has also been identified during January in the State of Sao Paulo. Despite amino acid changes and changes in potential glycosylation motifs, 6B.1 viruses were well inhibited by the reference ferret antiserum against A/California/07/2009 virus, the $\mathrm{A}(\mathrm{H} 1 \mathrm{~N} 1) \mathrm{pdm} 09$ component of the vaccine for the 2016 influenza season.

KEYWORDS: Influenza. National Influenza Surveillance Network. Molecular epidemiology. Vaccination strategy. Public health.

Compared to previous years, seasonal influenza activity commenced early in São Paulo State, Brazil, Southern hemisphere. Influenza A(H1N1)pdm09 virus detection started in January 2016 during the summer season with hot temperatures, and it was the predominating strain in autumn ${ }^{1,2}$. In contrast, influenza seasonal activity commenced late in some countries in Western Europe, North America and Eastern Asia. Based on the WHO global influenza surveillance, in countries with influenza $\mathrm{A}(\mathrm{H} 1 \mathrm{~N} 1) \mathrm{pdm} 09$ virus predominance, the hospitalization and intensive care unit (ICU) admission patterns seem to be similar to previous seasons when this virus predominated and young/middle-aged adults experienced severe disease ${ }^{3}$. The aim of this study was to investigate the genetic pattern of influenza A(H1N1)pdm09 in the São Paulo State.

A total of 479 respiratory samples, collected in January by Sentinel Surveillance Units, were screened by real-time RT-PCR (qRT - PCR) ${ }^{4}$. Among them, 30 Influenza virus $\mathrm{A}(\mathrm{H} 1 \mathrm{~N} 1) \mathrm{pdm} 09$ presenting ct values $\leq 30$ were identified. A total of 6 viruses were sequenced by using an Applied Biosystems BigDye ${ }^{\circledR}$ Terminator v3.1 Cycle Sequencing Kit with reaction products resolved on an Applied Biosystems Sequencer 3730 DNA Analyzer. Nucleotide sequences were aligned using MUSCLE 5 . Sequences alignment results were further analyzed using the BioEdit program ${ }^{6}$.

Our Institution is one of the National Influenza Centers accredited by the World Health Organization. The present follow up study of influenza surveillance has been 
approved by the Conselho Nacional de Ética em Pesquisa (CONEP) $-135 / 2002$.

\section{Phylogenetic analyses}

The TREESUB phylogenetic program (available from https://github.com/tamuri/treesub) was used to estimate the maximum likelihood phylogenetic trees using RAxML and PAML, followed by branch annotation of amino acid substitutions. The general time reversible $+\Gamma$ (GTR+GAMMA) nucleotide substitution model was selected in RAxML v.7.3.0 for tree inference ${ }^{7}$. Ancestral codon substitutions for each gene were estimated using baseml, as implemented in $\mathrm{PAML}^{8}$ using the ML trees inferred. Nonsynonymous substitutions were then transcribed onto the consensus gene phylogenies and visualized in Figure 1 Tree v1.4.2 (available from http:// tree.bio.ed.ac.uk/software/figtree/).

\section{Evolutionary Relationships Among Influenza A(H1N1)pdm09 Hemagglutinin (HA) Gene}

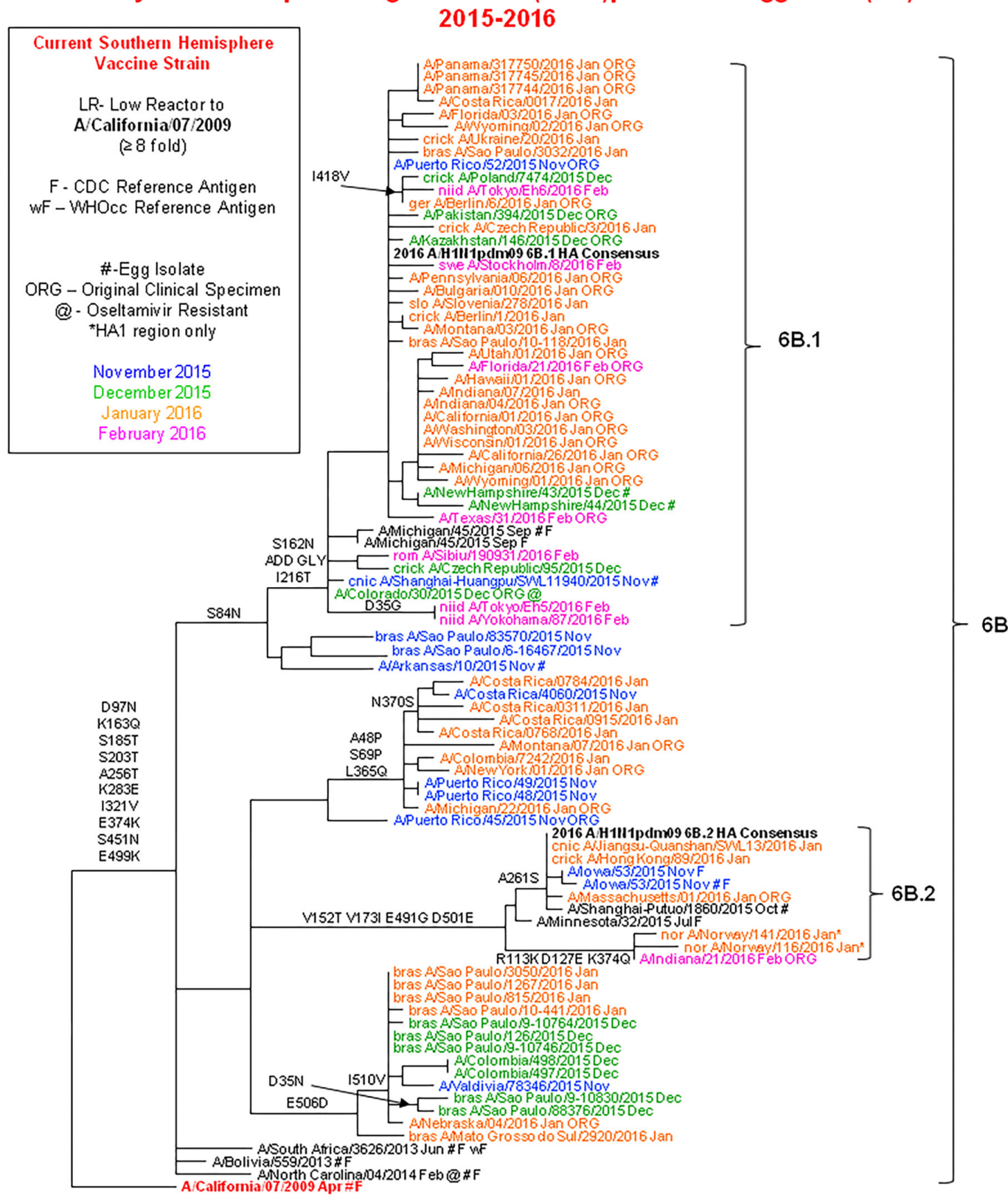

Figure 1 - Molecular epidemiology of influenza A(H1N1)pdm09 hemagglutinin gene circulating in São Paulo, state, Brazil: 2016 anticipated influenza season. 
Influenza $\mathrm{A}(\mathrm{H} 1 \mathrm{~N} 1)$ pdm09 viruses identified in São Paulo State during November 2015 belong to the 6B genetic group, presenting AA changes in the $\mathrm{HA}$ at residue E179D and I183V in the HA2 region. Figure 1 shows two viruses from Brazil collected in January that belong to the new 6B.1 subgroup (A/Sao Paulo/10-118/2016 and A/Sao Paulo/3032/2016). The HA sequences from São Paulo State obtained in this study, which were also used in the phylogenetic analysis, were deposited in the EpiFlu database of the Global Initiative on sharing Avian Influenza Data (GISAID) under the following accession numbers: EPI725841, EPI725838.

Influenza $\mathrm{A}(\mathrm{H} 1 \mathrm{~N} 1) \mathrm{pdm} 09$ group 6B has also been identified during January in São Paulo State as shown in Figure 1, which were also used in the phylogenetic analysis; A/Sao Paulo/3050/2016; A/Sao Paulo/1267/2016; A/Sao Paulo/815/2016; A/Sao Paulo/10-441/2016 deposited in the GISAID under the follow accession numbers EPI725839, EPI725844, EPI704027, EPI725842, respectively.

The phylogenetic analysis of the A(H1N1)pdm09 HA gene demonstrated that $\mathrm{HA}$ genes of the recent viruses diverged into genetic groups $6 \mathrm{~A}, 6 \mathrm{~B}$ or $6 \mathrm{C}$, with all viruses collected since September $1^{\text {st }}, 2015$ belonging to the genetic subgroup 6B. Subgroups $6 \mathrm{~B}$ and $6 \mathrm{C}$ share AA changes in the HA at residues K283E and E499K (mature A(H1N1) pdm09 numbering after the signal peptide). Subgroup 6B viruses also possess AA changes in the $\mathrm{HA}$ at residues K163Q and A256T. Within subgroup 6B, additional subgroups with shared amino acid changes have emerged. The majority of viruses share an AA change at residue S84N; among these more than half share two additional changes at residues $\mathrm{S} 162 \mathrm{~N}$ (adds a glycosylation motif) and I216T (new subgroup 6B.1). A smaller group of 6B viruses (new subgroup 6B.2) shares AA changes at residues V152T, V173I, E491G and D501E9

Despite amino acid changes and changes in glycosylation, 6B.1 viruses were well inhibited by the reference ferret antiserum against $\mathrm{A} / \mathrm{California} / 07 / 2009$ virus the $\mathrm{A}(\mathrm{H} 1 \mathrm{~N} 1)$ pdm09 component of the vaccine for 2016 influenza season ${ }^{9}$.

In addition, a pool of human post-vaccination sera collected from healthy adults in the United States of America who received influenza vaccine in the 2015-2016 seasons well inhibited all recent viruses tested in the WHO $\mathrm{CC}$ at the Centers for Disease Control and Prevention (CDC) in Atlanta ${ }^{9}$

According to the literature data, the United Kingdom (UK), as others countries in Europe, has experienced a season dominated by circulation of influenza $A(H 1 N 1)$ pdm09 with reports of increases in hospitalizations and ICU admissions mainly in younger adults. The epidemiological observations are consistent with earlier seasons in the UK dominated by circulation of $\mathrm{A}(\mathrm{H} 1 \mathrm{~N} 1) \mathrm{pdm} 09$, in particular in 2010/11, the first post-pandemic season ${ }^{10}$. In São Paulo State this influenza virus $\mathrm{A}(\mathrm{H} 1 \mathrm{~N} 1) \mathrm{pdm} 09$ pattern of seasonality occurred in 2013 (personnel communication), and in the currently anticipated influenza virus season 2016, influenza $\mathrm{A}(\mathrm{H} 1 \mathrm{~N} 1)$ pdm09 predominated.

Influenza virological surveillance follow-up will provide the antigenic and phylogenetic patterns of influenza $\mathrm{A}(\mathrm{H} 1 \mathrm{~N} 1)$ pdm09 virus circulation during the coming winter and early spring period in São Paulo State. Taking into account that Brazilian vacation period corresponds to the North America and Western Europe influenza season (January/February), and also considering the late influenza virus seasonality in these regions, this pattern of seasonality may be considered to interpret the earlier influenza seasonality in São Paulo State in 2016.

\section{ACKNOWLEDGEMENTS}

Our gratitude to the personnel of the Biological Sample Management Center/Institute Adolfo Lutz; Nilva Matias dos Reis, Ana Lúcia de Carvalho Avelino and Márcia Theobaldo for laboratorial technique support.

We also would like to thank sentinel nurses, practitioners and physicians of the Sentinel Surveillance Units of São Paulo State, Brazil, who provided quantitative information and collected samples from patients; the personnel from the Epidemiological Surveillance Centre, State Secretariat for Health of São Paulo, SP, Brazil; Municipal and State Secretariat for Health of São Paulo, SP, Brazil; and the personnel of the Ministry of Health of Brazil.

\section{REFERENCES}

1. Brasil. Ministério da Saúde. Situação epidemiológica/Dados. Brasília: Ministério da Saúde; 2016. [cited 2016 Mar 16]. Available from: http://portalsaude.saude.gov.br/index.php/ situacao-epidemiologica-dados-influenza

2. São Paulo. Secretaria da Saúde. Coordenadoria de Controle de Doenças. Centro de Vigilância Epidemiológica "Prof. Alexandre Vranjac". Informe técnico: situação epidemiológica da influenza. São Paulo: Secretaria da Saúde; 2016. [cited 2016 Aug 31]. Available from: http://www.cremesp.org.br/pdfs/ Informe_tecnico_flu_17mar2016.pdf.

3. World Health Organization. Risk assessment: seasonal influenza A(H1N1)pdm09. Geneva: WHO; 2016 [cited 2016 Feb 08]. Available from: http://www.who.int/influenza/publications/ riskassessment_AH1N1pdm09_201602/en/

4. Shu B, Wu KH, Emery S, Villanueva J, Johnson R, Guthrie $\mathrm{E}$, et al. Design and performance of the $\mathrm{CDC}$ real-time 
reverse transcriptase PCR swine flu panel for detection of 2009 A (H1N1) pandemic influenza virus. J Clin Microbiol. 2011;49:2614-9.

5. Edgar RC. MUSCLE: multiple sequence alignment with high accuracy and high throughput. Nucleic Acid Res. 2004;32:1792-7.

6. Hall TA. BioEdit: a user-friendly biological sequence alignment editor and analysis program for Windows 95/98/NT. Nucl Acids Symp Ser. 1999;41:95-8.

7. Stamatakis A. RAxML-VI-HPC: maximum likelihood-based phylogenetic analyses with thousands of taxa and mixed models. Bioinformatics. 2006;22:2688-90.
8. Yang Z. PAML 4: phylogenetic analysis by maximum likelihood. Mol Biol Evol. 2007;24:1586-91.

9. Recommended composition of influenza virus vaccines for use in the 2016-2017 northern hemisphere influenza season. Wkly Epidemiol Rec. 2016;91:121-32.

10. Pebody R, Warburton F, Ellis J, Andrews N, Potts A, Cottrell S, et al. Effectiveness of seasonal influenza vaccine in preventing laboratory-confirmed influenza in primary care in the United Kingdom: 2015/16 mid-season results. Euro Surveill. 2016; 21

Supplemental Table - Origin of the haemaglutinin sequences of influenza $A(\mathrm{H} 1 \mathrm{~N} 1)$ pdm09 isolates for the phylogenetic analysis . We acknowledge the authors, originating and submitting laboratories of the sequences from GISAID's EpiFlu ${ }^{\mathrm{TM}}$ Database on which this research is based. The list is detailed below

\begin{tabular}{|c|c|c|c|c|c|c|c|}
\hline Segment ID & Segment & Country & Collection date & Isolate name & Originating Lab & Submitting Lab & Authors \\
\hline EPI691137 & $\mathrm{HA}$ & United States & 2015-Nov-05 & A/Arkansas/10/2015 & $\begin{array}{c}\text { Arkansas Children's } \\
\text { Hospital }\end{array}$ & $\begin{array}{c}\text { Centers for Disease } \\
\text { Control and } \\
\text { Prevention }\end{array}$ & \\
\hline EPI706882 & $\mathrm{HA}$ & Germany & 2016-Jan-11 & A/Berlin/1/2016 & $\begin{array}{l}\text { Robert-Koch- } \\
\text { Institute }\end{array}$ & $\begin{array}{l}\text { Crick Worldwide } \\
\text { Influenza Centre }\end{array}$ & \\
\hline EPI733770 & $\mathrm{HA}$ & Germany & 2016-Jan-19 & A/Berlin/6/2016 & $\begin{array}{l}\text { Robert-Koch- } \\
\text { Institute }\end{array}$ & $\begin{array}{l}\text { Crick Worldwide } \\
\text { Influenza Centre }\end{array}$ & \\
\hline EPI465091 & $\mathrm{HA}$ & $\begin{array}{c}\text { Bolivia, } \\
\text { Plurinationial } \\
\text { State of }\end{array}$ & 2013-Jun-08 & A/Bolivia/559/2013 & $\begin{array}{c}\text { Instituto Nacional } \\
\text { de Laboratoriosde } \\
\text { Salud (INLASA) }\end{array}$ & $\begin{array}{c}\text { Centers for Disease } \\
\text { Control and } \\
\text { Prevention }\end{array}$ & \\
\hline EPI700599 & $\mathrm{HA}$ & Bulgaria & 2016-Jan-08 & A/Bulgaria/010/2016 & $\begin{array}{l}\text { National Influenza } \\
\text { Laboratory, Natl. } \\
\text { Center of Inf. \& } \\
\text { Parasitic Dis }\end{array}$ & $\begin{array}{c}\text { Centers for Disease } \\
\text { Control and } \\
\text { Prevention }\end{array}$ & \\
\hline EPI700615 & $\mathrm{HA}$ & United States & 2016-Jan-04 & A/California/01/2016 & $\begin{array}{c}\text { California } \\
\text { Department of } \\
\text { Health Services }\end{array}$ & $\begin{array}{c}\text { Centers for Disease } \\
\text { Control and } \\
\text { Prevention }\end{array}$ & \\
\hline EPI176620 & $\mathrm{HA}$ & United States & 2009-Apr-09 & A/California/07/2009 & $\begin{array}{c}\text { Naval Health } \\
\text { Research Center }\end{array}$ & $\begin{array}{c}\text { Centers for Disease } \\
\text { Control and } \\
\text { Prevention }\end{array}$ & \\
\hline EPI717170 & $\mathrm{HA}$ & United States & 2016-Jan-08 & A/California/26/2016 & $\begin{array}{c}\text { California } \\
\text { Department of } \\
\text { Health Services }\end{array}$ & $\begin{array}{c}\text { Centers for Disease } \\
\text { Control and } \\
\text { Prevention }\end{array}$ & \\
\hline EPI725887 & $\mathrm{HA}$ & Colombia & 2015-Dec-10 & A/Colombia/497/2015 & $\begin{array}{l}\text { Instituto Nacional } \\
\text { de Salud de } \\
\text { Columbia }\end{array}$ & $\begin{array}{c}\text { Centers for Disease } \\
\text { Control and } \\
\text { Prevention }\end{array}$ & \\
\hline EPI725895 & $\mathrm{HA}$ & Colombia & 2015-Dec-10 & A/Colombia/498/2015 & $\begin{array}{l}\text { Instituto Nacional } \\
\text { de Salud de } \\
\text { Columbia }\end{array}$ & $\begin{array}{c}\text { Centers for Disease } \\
\text { Control and } \\
\text { Prevention }\end{array}$ & \\
\hline EPI725911 & $\mathrm{HA}$ & Colombia & 2016-Jan-01 & A/Colombia/7242/2016 & $\begin{array}{c}\text { Instituto Nacional } \\
\text { de Salud de } \\
\text { Columbia } \\
\end{array}$ & $\begin{array}{c}\text { Centers for Disease } \\
\text { Control and } \\
\text { Prevention }\end{array}$ & \\
\hline EPI700671 & $\mathrm{HA}$ & United States & 2015-Dec-14 & A/Colorado/30/2015 & $\begin{array}{l}\text { Colorado } \\
\text { Department of } \\
\text { Health Lab }\end{array}$ & $\begin{array}{c}\text { Centers for Disease } \\
\text { Control and } \\
\text { Prevention }\end{array}$ & \\
\hline EPI706013 & $\mathrm{HA}$ & Costa Rica & 2016-Jan-04 & A/Costa Rica/0017/2016 & $\begin{array}{c}\text { Laboratorio } \\
\text { Nacional de } \\
\text { Influenza }\end{array}$ & $\begin{array}{c}\text { Centers for Disease } \\
\text { Control and } \\
\text { Prevention }\end{array}$ & \\
\hline EPI716691 & $\mathrm{HA}$ & Costa Rica & 2016-Jan-08 & A/Costa Rica/0311/2016 & $\begin{array}{l}\text { Laboratorio } \\
\text { Nacional de } \\
\text { Influenza }\end{array}$ & $\begin{array}{c}\text { Centers for Disease } \\
\text { Control and } \\
\text { Prevention }\end{array}$ & \\
\hline EPI706021 & $\mathrm{HA}$ & Costa Rica & 2016-Jan-08 & A/Costa Rica/0768/2016 & $\begin{array}{c}\text { Laboratorio } \\
\text { Nacional de } \\
\text { Influenza }\end{array}$ & $\begin{array}{c}\text { Centers for Disease } \\
\text { Control and } \\
\text { Prevention }\end{array}$ & \\
\hline
\end{tabular}


Supplemental Table - Origin of the haemaglutinin sequences of influenza $A(H 1 N 1) p d m 09$ isolates for the phylogenetic analysis . We acknowledge the authors, originating and submitting laboratories of the sequences from GISAID's EpiFlu ${ }^{\mathrm{TM}}$ Database on which this research is based. The list is detailed below (cont.)

\begin{tabular}{|c|c|c|c|c|c|c|c|}
\hline Segment ID & Segment & Country & Collection date & Isolate name & Originating Lab & Submitting Lab & Authors \\
\hline EPI706029 & $\mathrm{HA}$ & Costa Rica & 2016-Jan-08 & A/Costa Rica/0784/2016 & $\begin{array}{l}\text { Laboratorio } \\
\text { Nacional de } \\
\text { Influenza }\end{array}$ & $\begin{array}{c}\text { Centers for Disease } \\
\text { Control and } \\
\text { Prevention }\end{array}$ & \\
\hline EPI716699 & $\mathrm{HA}$ & Costa Rica & 2016-Jan-09 & A/Costa Rica/0915/2016 & $\begin{array}{l}\text { Laboratorio } \\
\text { Nacional de } \\
\text { Influenza }\end{array}$ & $\begin{array}{c}\text { Centers for Disease } \\
\text { Control and } \\
\text { Prevention }\end{array}$ & \\
\hline EPI690877 & $\mathrm{HA}$ & Costa Rica & 2015-Nov-20 & A/Costa Rica/4060/2015 & $\begin{array}{l}\text { Laboratorio } \\
\text { Nacional de } \\
\text { Influenza }\end{array}$ & $\begin{array}{c}\text { Centers for Disease } \\
\text { Control and } \\
\text { Prevention }\end{array}$ & \\
\hline EPI706894 & $\mathrm{HA}$ & $\begin{array}{c}\text { Czech } \\
\text { Republic }\end{array}$ & 2016-Jan-01 & A/Czech Republic/3/2016 & $\begin{array}{l}\text { National Institute of } \\
\text { Public Health }\end{array}$ & $\begin{array}{l}\text { Crick Worldwide } \\
\text { Influenza Centre }\end{array}$ & \\
\hline EPI706896 & $\mathrm{HA}$ & $\begin{array}{c}\text { Czech } \\
\text { Republic }\end{array}$ & 2015-Dec-01 & A/Czech Republic/95/2015 & $\begin{array}{l}\text { National Institute of } \\
\text { Public Health }\end{array}$ & $\begin{array}{l}\text { Crick Worldwide } \\
\text { Influenza Centre }\end{array}$ & \\
\hline EPI709468 & $\mathrm{HA}$ & United States & 2016-Jan-13 & A/Florida/03/2016 & $\begin{array}{c}\text { Florida Department } \\
\text { of Health- } \\
\text { Jacksonville }\end{array}$ & $\begin{array}{c}\text { Centers for Disease } \\
\text { Control and } \\
\text { Prevention }\end{array}$ & \\
\hline EPI726014 & $\mathrm{HA}$ & United States & 2016-Feb-19 & A/Florida/21/2016 & $\begin{array}{c}\text { Florida Department } \\
\text { of Health-Tampa }\end{array}$ & $\begin{array}{c}\text { Centers for Disease } \\
\text { Control and } \\
\text { Prevention }\end{array}$ & \\
\hline EPI706061 & $\mathrm{HA}$ & United States & 2016-Jan-12 & A/Hawaii/01/2016 & $\begin{array}{c}\text { State of Hawaii } \\
\text { Department of } \\
\text { Health }\end{array}$ & $\begin{array}{c}\text { Centers for Disease } \\
\text { Control and } \\
\text { Prevention }\end{array}$ & \\
\hline EPI697711 & $\mathrm{HA}$ & $\begin{array}{l}\text { Hong Kong } \\
\text { (SAR) }\end{array}$ & 2016-Jan-04 & A/Hong Kong/89/2016 & $\begin{array}{c}\text { Government Virus } \\
\text { Unit }\end{array}$ & $\begin{array}{l}\text { Crick Worldwide } \\
\text { Influenza Centre }\end{array}$ & \\
\hline EPI732918 & $\mathrm{HA}$ & United States & 2016-Jan-25 & A/Indiana/04/2016 & $\begin{array}{c}\text { Indiana State } \\
\text { Department of } \\
\text { Health Laboratories }\end{array}$ & $\begin{array}{c}\text { Centers for Disease } \\
\text { Control and } \\
\text { Prevention } \\
\end{array}$ & \\
\hline EPI715972 & $\mathrm{HA}$ & United States & 2016-Jan-24 & A/Indiana/07/2016 & $\begin{array}{c}\text { Indiana State } \\
\text { Department of } \\
\text { Health Laboratories }\end{array}$ & $\begin{array}{c}\text { Centers for Disease } \\
\text { Control and } \\
\text { Prevention }\end{array}$ & \\
\hline EPI717210 & $\mathrm{HA}$ & United States & 2016-Feb-04 & A/Indiana/21/2016 & $\begin{array}{c}\text { Indiana State } \\
\text { Department of } \\
\text { Health Laboratories }\end{array}$ & $\begin{array}{c}\text { Centers for Disease } \\
\text { Control and } \\
\text { Prevention }\end{array}$ & \\
\hline EPI709638 & $\mathrm{HA}$ & United States & 2015-Nov-04 & A/lowa/53/2015 & $\begin{array}{c}\text { lowa State Hygienic } \\
\text { Laboratory }\end{array}$ & $\begin{array}{c}\text { Centers for Disease } \\
\text { Control and } \\
\text { Prevention }\end{array}$ & \\
\hline EPI709630 & $\mathrm{HA}$ & United States & 2015-Nov-04 & A/lowa/53/2015 & $\begin{array}{c}\text { lowa State Hygienic } \\
\text { Laboratory }\end{array}$ & $\begin{array}{c}\text { Centers for Disease } \\
\text { Control and } \\
\text { Prevention }\end{array}$ & \\
\hline EPI697117 & $\mathrm{HA}$ & China & 2016-Jan-05 & $\begin{array}{l}\text { A/Jiangsu-Quanshan/ } \\
\text { SWL13/2016 }\end{array}$ & $\begin{array}{c}\text { WHO Chinese } \\
\text { National Influenza } \\
\text { Center }\end{array}$ & $\begin{array}{c}\text { WHO Chinese } \\
\text { National Influenza } \\
\text { Center }\end{array}$ & $\begin{array}{c}\text { Yu Lan,Weijuan Huang, } \\
\text { Xiyan Li, Xiang Zhao, } \\
\text { Yanhui Cheng, Minju } \\
\text { Tan, Dayan Wang, } \\
\text { Yuelong Shu }\end{array}$ \\
\hline EPI691262 & $\mathrm{HA}$ & Kazakhstan & 2015-Dec-02 & A/Kazakhstan/146/2015 & CSEE & $\begin{array}{c}\text { Centers for Disease } \\
\text { Control and } \\
\text { Prevention }\end{array}$ & \\
\hline EPI700788 & $\mathrm{HA}$ & United States & 2016-Jan-11 & A/Massachusetts/01/2016 & $\begin{array}{l}\text { Massachusetts } \\
\text { Department of } \\
\text { Public Health }\end{array}$ & $\begin{array}{c}\text { Centers for Disease } \\
\text { Control and } \\
\text { Prevention }\end{array}$ & \\
\hline EPI725840 & $\mathrm{HA}$ & Brazil & 2016-Jan-18 & $\begin{array}{l}\text { A/Mato Grosso do } \\
\text { Sul/2920/2016 }\end{array}$ & Instituto Adolfo Lutz & Instituto Adolfo Lutz & $\begin{array}{c}\text { Santos, Katia; Silva, } \\
\text { Daniela; Benega, } \\
\text { Margarete; Santos, } \\
\text { Cecilia; Paiva, Terezinha }\end{array}$ \\
\hline EPI732896 & $\mathrm{HA}$ & United States & 2016-Jan-19 & A/Michigan/06/2016 & $\begin{array}{l}\text { University of } \\
\text { Michigan SPH } \\
\text { EPID }\end{array}$ & $\begin{array}{c}\text { Centers for Disease } \\
\text { Control and } \\
\text { Prevention }\end{array}$ & \\
\hline EPI717234 & $\mathrm{HA}$ & United States & 2016-Jan-04 & A/Michigan/22/2016 & $\begin{array}{c}\text { Michigan } \\
\text { Department of } \\
\text { Community Health }\end{array}$ & $\begin{array}{c}\text { Centers for Disease } \\
\text { Control and } \\
\text { Prevention }\end{array}$ & \\
\hline EPI699812 & $\mathrm{HA}$ & United States & 2015-Sep-07 & A/Michigan/45/2015 & $\begin{array}{c}\text { Michigan } \\
\text { Department of } \\
\text { Community Health }\end{array}$ & $\begin{array}{c}\text { Centers for Disease } \\
\text { Control and } \\
\text { Prevention }\end{array}$ & \\
\hline
\end{tabular}


Supplemental Table - Origin of the haemaglutinin sequences of influenza A(H1N1)pdm09 isolates for the phylogenetic analysis . We acknowledge the authors, originating and submitting laboratories of the sequences from GISAID's EpiFlu ${ }^{\mathrm{TM}}$ Database on which this research is based. The list is detailed below (cont.)

\begin{tabular}{|c|c|c|c|c|c|c|c|}
\hline Segment ID & Segment & Country & Collection date & Isolate name & Originating Lab & Submitting Lab & Authors \\
\hline EPI685579 & $\mathrm{HA}$ & United States & 2015-Sep-07 & A/Michigan/45/2015 & $\begin{array}{c}\text { Michigan } \\
\text { Department of } \\
\text { Community Health } \\
\end{array}$ & $\begin{array}{c}\text { Centers for Disease } \\
\text { Control and } \\
\text { Prevention } \\
\end{array}$ & \\
\hline EPI699804 & $\mathrm{HA}$ & United States & 2015-Jul-09 & A/Minnesota/32/2015 & $\begin{array}{c}\text { Minnesota } \\
\text { Department of } \\
\text { Health } \\
\end{array}$ & $\begin{array}{c}\text { Centers for Disease } \\
\text { Control and } \\
\text { Prevention } \\
\end{array}$ & \\
\hline EPI706181 & $\mathrm{HA}$ & United States & 2016-Jan-09 & A/Montana/03/2016 & $\begin{array}{c}\text { Montana Laboratory } \\
\text { Services Bureau }\end{array}$ & $\begin{array}{c}\text { Centers for Disease } \\
\text { Control and } \\
\text { Prevention }\end{array}$ & \\
\hline EPI709516 & $\mathrm{HA}$ & United States & 2016-Jan-10 & A/Montana/07/2016 & $\begin{array}{l}\text { Montana Laboratory } \\
\text { Services Bureau }\end{array}$ & $\begin{array}{c}\text { Centers for Disease } \\
\text { Control and } \\
\text { Prevention }\end{array}$ & \\
\hline EPI716444 & $\mathrm{HA}$ & United States & 2016-Jan-21 & A/Nebraska/04/2016 & $\begin{array}{c}\text { Nebraska Public } \\
\text { Health Lab }\end{array}$ & $\begin{array}{c}\text { Centers for Disease } \\
\text { Control and } \\
\text { Prevention }\end{array}$ & \\
\hline EPI695421 & $\mathrm{HA}$ & United States & 2015-Dec-03 & A/New Hampshire/43/2015 & $\begin{array}{l}\text { New Hampshire } \\
\text { Public Health } \\
\text { Laboratories }\end{array}$ & $\begin{array}{c}\text { Centers for Disease } \\
\text { Control and } \\
\text { Prevention }\end{array}$ & \\
\hline EPI695469 & $\mathrm{HA}$ & United States & 2015-Dec-04 & A/New Hampshire/44/2015 & $\begin{array}{l}\text { New Hampshire } \\
\text { Public Health } \\
\text { Laboratories }\end{array}$ & $\begin{array}{c}\text { Centers for Disease } \\
\text { Control and } \\
\text { Prevention }\end{array}$ & \\
\hline EPI700817 & $\mathrm{HA}$ & United States & 2016-Jan-05 & A/New York/01/2016 & $\begin{array}{l}\text { New York City } \\
\text { Department of } \\
\text { Health }\end{array}$ & $\begin{array}{c}\text { Centers for Disease } \\
\text { Control and } \\
\text { Prevention }\end{array}$ & \\
\hline EPI541854 & $\mathrm{HA}$ & United States & 2014-Feb-16 & A/North Carolina/04/2014 & $\begin{array}{l}\text { Mission Hospital } \\
\text { Microbiology Lab }\end{array}$ & $\begin{array}{c}\text { Centers for Disease } \\
\text { Control and } \\
\text { Prevention } \\
\end{array}$ & \\
\hline EPI695331 & $\mathrm{HA}$ & Norway & 2016-Jan-04 & A/Norway/116/2016 & $\begin{array}{l}\text { Drammen Hospital } \\
\text { / Vestreviken HF, } \\
\text { Depto for Medical } \\
\text { Microbiology } \\
\text { section Drammen }\end{array}$ & $\begin{array}{l}\text { Norwegian Institute of } \\
\text { Public Health }\end{array}$ & $\begin{array}{c}\text { Bragstad, K; Dudman, } \\
\text { SG; Waalen, K; } \\
\text { Hungnes, O }\end{array}$ \\
\hline EPI695336 & $\mathrm{HA}$ & Norway & 2016-Jan-04 & A/Norway/141/2016 & $\begin{array}{c}\text { Unilabs Telelab, } \\
\text { Laboratory } \\
\text { for Medical } \\
\text { Microbiology }\end{array}$ & $\begin{array}{l}\text { Norwegian Institute of } \\
\text { Public Health }\end{array}$ & $\begin{array}{c}\text { Bragstad, K; Dudman, } \\
\text { SG; Waalen, K; } \\
\text { Hungnes, O }\end{array}$ \\
\hline EPI706259 & $\mathrm{HA}$ & Pakistan & 2015-Dec-01 & A/Pakistan/394/2015 & $\begin{array}{c}\text { National Institute of } \\
\text { Health }\end{array}$ & $\begin{array}{c}\text { Centers for Disease } \\
\text { Control and } \\
\text { Prevention }\end{array}$ & \\
\hline EPI716460 & $\mathrm{HA}$ & Panama & 2016-Jan-09 & A/Panama/317744/2016 & $\begin{array}{c}\text { Instituto } \\
\text { Conmemorativo } \\
\text { Gorgas de Estudios } \\
\text { de la Salud }\end{array}$ & $\begin{array}{l}\text { Centers for Disease } \\
\text { Control and } \\
\text { Prevention }\end{array}$ & \\
\hline EPI716468 & $\mathrm{HA}$ & Panama & 2016-Jan-09 & A/Panama/317745/2016 & $\begin{array}{c}\text { Instituto } \\
\text { Conmemorativo } \\
\text { Gorgas de Estudios } \\
\text { de la Salud }\end{array}$ & $\begin{array}{l}\text { Centers for Disease } \\
\text { Control and } \\
\text { Prevention }\end{array}$ & \\
\hline EPI716476 & $\mathrm{HA}$ & Panama & 2016-Jan-12 & A/Panama/317750/2016 & $\begin{array}{c}\text { Instituto } \\
\text { Conmemorativo } \\
\text { Gorgas de Estudios } \\
\text { de la Salud }\end{array}$ & $\begin{array}{c}\text { Centers for Disease } \\
\text { Control and } \\
\text { Prevention }\end{array}$ & \\
\hline EPI709524 & $\mathrm{HA}$ & United States & 2016-Jan-05 & A/Pennsylvania/06/2016 & $\begin{array}{l}\text { Pennsylvania } \\
\text { Department of } \\
\text { Health }\end{array}$ & $\begin{array}{c}\text { Centers for Disease } \\
\text { Control and } \\
\text { Prevention }\end{array}$ & \\
\hline EPI697742 & $\mathrm{HA}$ & Poland & 2015-Dec-31 & A/Poland/7474/2015 & $\begin{array}{c}\text { National Institute } \\
\text { of Public Health - } \\
\text { National Institute of } \\
\text { Hygiene }\end{array}$ & $\begin{array}{l}\text { Crick Worldwide } \\
\text { Influenza Centre }\end{array}$ & \\
\hline EPI716012 & $\mathrm{HA}$ & Puerto Rico & 2015-Nov-15 & A/Puerto Rico/45/2015 & $\begin{array}{c}\text { Puerto Rico } \\
\text { Department of } \\
\text { Health }\end{array}$ & $\begin{array}{c}\text { Centers for Disease } \\
\text { Control and } \\
\text { Prevention }\end{array}$ & \\
\hline EPI706290 & $\mathrm{HA}$ & Puerto Rico & 2015-Nov-18 & A/Puerto Rico/48/2015 & $\begin{array}{l}\text { Puerto Rico } \\
\text { Department of } \\
\text { Health }\end{array}$ & $\begin{array}{c}\text { Centers for Disease } \\
\text { Control and } \\
\text { Prevention }\end{array}$ & \\
\hline
\end{tabular}


Supplemental Table - Origin of the haemaglutinin sequences of influenza $A(H 1 N 1) p d m 09$ isolates for the phylogenetic analysis . We acknowledge the authors, originating and submitting laboratories of the sequences from GISAID's EpiFlu ${ }^{\mathrm{TM}}$ Database on which this research is based. The list is detailed below (cont.)

\begin{tabular}{|c|c|c|c|c|c|c|c|}
\hline Segment ID & Segment & Country & Collection date & Isolate name & Originating Lab & Submitting Lab & Authors \\
\hline EPI710546 & $\mathrm{HA}$ & Puerto Rico & 2015-Nov-20 & A/Puerto Rico/49/2015 & $\begin{array}{c}\text { Puerto Rico } \\
\text { Department of } \\
\text { Health }\end{array}$ & $\begin{array}{c}\text { Centers for Disease } \\
\text { Control and } \\
\text { Prevention }\end{array}$ & \\
\hline EPI716020 & $\mathrm{HA}$ & Puerto Rico & 2015-Nov-27 & A/Puerto Rico/52/2015 & $\begin{array}{l}\text { Puerto Rico } \\
\text { Department of } \\
\text { Health }\end{array}$ & $\begin{array}{c}\text { Centers for Disease } \\
\text { Control and } \\
\text { Prevention } \\
\end{array}$ & \\
\hline EPI725841 & $\mathrm{HA}$ & Brazil & 2016-Jan-04 & A/Sao Paulo/10-118/2016 & Instituto Adolfo Lutz & Instituto Adolfo Lutz & $\begin{array}{c}\text { Santos, Katia; Silva, } \\
\text { Daniela; Benega, } \\
\text { Margarete; Santos, } \\
\text { Cecilia; Paiva, Terezinha }\end{array}$ \\
\hline EPI725842 & $\mathrm{HA}$ & Brazil & 2016-Jan-11 & A/Sao Paulo/10-441/2016 & Instituto Adolfo Lutz & Instituto Adolfo Lutz & $\begin{array}{c}\text { Santos, Katia; Silva, } \\
\text { Daniela; Benega, } \\
\text { Margarete; Santos, } \\
\text { Cecilia; Paiva, Terezinha }\end{array}$ \\
\hline EPI704025 & $\mathrm{HA}$ & Brazil & 2015-Dec-29 & A/Sao Paulo/126/2016 & Instituto Adolfo Lutz & Instituto Adolfo Lutz & $\begin{array}{c}\text { Santos, Katia; Silva, } \\
\text { Daniela; Benega, } \\
\text { Margarete; Santos, } \\
\text { Cecilia; Paiva, Terezinha }\end{array}$ \\
\hline EPI704028 & $\mathrm{HA}$ & Brazil & 2016-Jan-11 & A/Sao Paulo/1267/2016 & Instituto Adolfo Lutz & Instituto Adolfo Lutz & $\begin{array}{c}\text { Santos, Katia; Silva, } \\
\text { Daniela; Benega, } \\
\text { Margarete; Santos, } \\
\text { Cecilia; Paiva, Terezinha }\end{array}$ \\
\hline EPI725838 & $\mathrm{HA}$ & Brazil & 2016-Jan-24 & A/Sao Paulo/3032/2016 & Instituto Adolfo Lutz & Instituto Adolfo Lutz & $\begin{array}{c}\text { Santos, Katia; Silva, } \\
\text { Daniela; Benega, } \\
\text { Margarete; Santos, } \\
\text { Cecilia; Paiva, Terezinha }\end{array}$ \\
\hline EPI725839 & $\mathrm{HA}$ & Brazil & 2016-Jan-25 & A/Sao Paulo/3050/2016 & Instituto Adolfo Lutz & Instituto Adolfo Lutz & $\begin{array}{c}\text { Santos, Katia; Silva, } \\
\text { Daniela; Benega, } \\
\text { Margarete; Santos, } \\
\text { Cecilia; Paiva, Terezinha }\end{array}$ \\
\hline EPI687384 & $\mathrm{HA}$ & Brazil & 2015-Nov-25 & A/Sao Paulo/6-16467/2015 & Instituto Adolfo Lutz & Instituto Adolfo Lutz & $\begin{array}{c}\text { Santos, Katia; Silva, } \\
\text { Daniela; Benega, } \\
\text { Margarete; Santos, } \\
\text { Cecilia; Paiva, Terezinha }\end{array}$ \\
\hline EPI704027 & $\mathrm{HA}$ & Brazil & 2016-Jan-08 & A/Sao Paulo/815/2016 & Instituto Adolfo Lutz & Instituto Adolfo Lutz & $\begin{array}{c}\text { Santos, Katia; Silva, } \\
\text { Daniela; Benega, } \\
\text { Margarete; Santos, } \\
\text { Cecilia; Paiva, Terezinha }\end{array}$ \\
\hline EPI685425 & $\mathrm{HA}$ & Brazil & 2015-Nov-20 & A/Sao Paulo/83570/2015 & Instituto Adolfo Lutz & Instituto Adolfo Lutz & $\begin{array}{c}\text { Santos, Katia; Silva, } \\
\text { Daniela; Benega, } \\
\text { Margarete; Santos, } \\
\text { Cecilia; Paiva, Terezinha }\end{array}$ \\
\hline EPI687387 & $\mathrm{HA}$ & Brazil & 2015-Dec-15 & A/Sao Paulo/88376/2015 & Instituto Adolfo Lutz & Instituto Adolfo Lutz & $\begin{array}{c}\text { Santos, Katia; Silva, } \\
\text { Daniela; Benega, } \\
\text { Margarete; Santos, } \\
\text { Cecilia; Paiva, Terezinha }\end{array}$ \\
\hline EPI687386 & $\mathrm{HA}$ & Brazil & 2015-Dec-07 & A/Sao Paulo/9-10746/2015 & Instituto Adolfo Lutz & Instituto Adolfo Lutz & $\begin{array}{c}\text { Santos, Katia; Silva, } \\
\text { Daniela; Benega, } \\
\text { Margarete; Santos, } \\
\text { Cecilia; Paiva, Terezinha }\end{array}$ \\
\hline EPI687385 & $\mathrm{HA}$ & Brazil & 2015-Dec-08 & A/Sao Paulo/9-10764/2015 & Instituto Adolfo Lutz & Instituto Adolfo Lutz & $\begin{array}{c}\text { Santos, Katia; Silva, } \\
\text { Daniela; Benega, } \\
\text { Margarete; Santos, } \\
\text { Cecilia; Paiva, Terezinha }\end{array}$ \\
\hline EPI704003 & $\mathrm{HA}$ & Brazil & 2015-Dec-17 & A/Sao Paulo/9-10830/2015 & Instituto Adolfo Lutz & Instituto Adolfo Lutz & $\begin{array}{c}\text { Santos, Katia; Silva, } \\
\text { Daniela; Benega, } \\
\text { Margarete; Santos, } \\
\text { Cecilia; Paiva, Terezinha }\end{array}$ \\
\hline EPI697225 & $\mathrm{HA}$ & China & 2015-Nov-20 & $\begin{array}{l}\text { A/Shanghai-Huangpu/ } \\
\text { SWL11940/2015 }\end{array}$ & $\begin{array}{l}\text { WHO Chinese } \\
\text { National Influenza } \\
\text { Center }\end{array}$ & $\begin{array}{c}\text { WHO Chinese } \\
\text { National Influenza } \\
\text { Center }\end{array}$ & $\begin{array}{c}\text { Yu Lan, Weijuan Huang, } \\
\text { Xiyan Li, Xiang Zhao, } \\
\text { Yanhui Cheng, Minju } \\
\text { Tan, Dayan Wang, } \\
\text { Yuelong Shu }\end{array}$ \\
\hline EPI709614 & $\mathrm{HA}$ & China & 2015-Oct-29 & A/Shanghai-Putuo/1860/2015 & $\begin{array}{l}\text { WHO Chinese } \\
\text { National Influenza } \\
\text { Center }\end{array}$ & $\begin{array}{c}\text { Centers for Disease } \\
\text { Control and } \\
\text { Prevention }\end{array}$ & \\
\hline
\end{tabular}


Supplemental Table - Origin of the haemaglutinin sequences of influenza $A(H 1 N 1) p d m 09$ isolates for the phylogenetic analysis . We acknowledge the authors, originating and submitting laboratories of the sequences from GISAID's EpiFlu ${ }^{\mathrm{TM}}$ Database on which this research is based. The list is detailed below (cont.)

\begin{tabular}{|c|c|c|c|c|c|c|c|}
\hline Segment ID & Segment & Country & Collection date & Isolate name & Originating Lab & Submitting Lab & Authors \\
\hline EPI711230 & $\mathrm{HA}$ & Romania & 2016-Feb-02 & A/Sibiu/190931/2016 & $\begin{array}{l}\text { Cantacuzino } \\
\text { Institute }\end{array}$ & Cantacuzino Institute & $\begin{array}{c}\text { Lazar, M.; Dinu, S.; } \\
\text { Oprea, M.; Necula, G.; } \\
\text { Ivanciuc, A.; Lupulescu, } \\
\text { E }\end{array}$ \\
\hline EPI709640 & $\mathrm{HA}$ & Slovenia & 2016-Jan-20 & A/Slovenia/278/2016 & & $\begin{array}{l}\text { National Laboratory } \\
\text { for Health } \\
\text { Environment and } \\
\text { Food }\end{array}$ & \\
\hline EPI577031 & $\mathrm{HA}$ & South Africa & 2013-Jun-06 & A/South Africa/3626/2013 & $\begin{array}{l}\text { National Institute for } \\
\text { Medical Research }\end{array}$ & $\begin{array}{c}\text { Centers for Disease } \\
\text { Control and } \\
\text { Prevention }\end{array}$ & \\
\hline EPI712265 & $\mathrm{HA}$ & Sweden & 2016-Feb-02 & A/Stockholm/8/2016 & & $\begin{array}{l}\text { Swedish Institute for } \\
\text { Infectious Disease } \\
\text { Control }\end{array}$ & \\
\hline EPI725683 & $\mathrm{HA}$ & United States & 2016-Feb-09 & A/Texas/31/2016 & $\begin{array}{l}\text { Texas Department } \\
\text { of State Health } \\
\text { Services-Laboratory } \\
\text { Services }\end{array}$ & $\begin{array}{l}\text { Centers for Disease } \\
\text { Control and } \\
\text { Prevention }\end{array}$ & \\
\hline EPI710931 & $\mathrm{HA}$ & Japan & 2016-Feb-01 & A/TOKYO/EH5/2016 & $\begin{array}{l}\text { National Institute of } \\
\text { Infectious Diseases } \\
\text { (NIID) }\end{array}$ & $\begin{array}{l}\text { National Institute of } \\
\text { Infectious Diseases } \\
\text { (NIID) }\end{array}$ & $\begin{array}{c}\text { Takashita, Emi; Fujisaki, } \\
\text { Seiichiro; Shirakura, } \\
\text { Masayuki; Watanabe, } \\
\text { Shinji; Odagiri, Takato }\end{array}$ \\
\hline EPI710939 & $\mathrm{HA}$ & Japan & 2016-Feb-02 & A/TOKYO/EH6/2016 & $\begin{array}{l}\text { National Institute of } \\
\text { Infectious Diseases } \\
\text { (NIID) }\end{array}$ & $\begin{array}{l}\text { National Institute of } \\
\text { Infectious Diseases } \\
\text { (NIID) }\end{array}$ & $\begin{array}{c}\text { Takashita, Emi; Fujisaki, } \\
\text { Seiichiro; Shirakura, } \\
\text { Masayuki; Watanabe, } \\
\text { Shinji; Odagiri, Takato }\end{array}$ \\
\hline EPI706970 & $\mathrm{HA}$ & Ukraine & 2016-Jan-03 & A/Ukraine/20/2016 & $\begin{array}{c}\text { Institute of } \\
\text { Epidemiology and } \\
\text { Infectious Diseases } \\
\text { AMS of Ukraine }\end{array}$ & $\begin{array}{l}\text { Crick Worldwide } \\
\text { Influenza Centre }\end{array}$ & \\
\hline EPI706298 & $\mathrm{HA}$ & United States & 2016-Jan-05 & $\mathrm{A} /$ Utah/01/2016 & $\begin{array}{c}\text { Utah Department of } \\
\text { Health }\end{array}$ & $\begin{array}{c}\text { Centers for Disease } \\
\text { Control and } \\
\text { Prevention } \\
\end{array}$ & \\
\hline EPI715948 & $\mathrm{HA}$ & Chile & 2015-Nov-02 & A/Valdivia/78346/2015 & $\begin{array}{l}\text { Instituto de Salud } \\
\text { Publica de Chile }\end{array}$ & $\begin{array}{c}\text { Centers for Disease } \\
\text { Control and } \\
\text { Prevention }\end{array}$ & \\
\hline EPI732837 & $\mathrm{HA}$ & United States & 2016-Jan-07 & A/Washington/03/2016 & $\begin{array}{c}\text { Marshfield } \\
\text { Clinic Research } \\
\text { Foundation }\end{array}$ & $\begin{array}{c}\text { Centers for Disease } \\
\text { Control and } \\
\text { Prevention }\end{array}$ & \\
\hline EPI698049 & $\mathrm{HA}$ & United States & 2016-Jan-08 & A/Wisconsin/01/2016 & $\begin{array}{c}\text { Wisconsin State } \\
\text { Laboratory of } \\
\text { Hygiene }\end{array}$ & $\begin{array}{c}\text { Centers for Disease } \\
\text { Control and } \\
\text { Prevention }\end{array}$ & \\
\hline EPI710586 & $\mathrm{HA}$ & United States & 2016-Jan-14 & A/Wyoming/01/2016 & $\begin{array}{l}\text { Wyoming Public } \\
\text { Health Laboratory }\end{array}$ & $\begin{array}{c}\text { Centers for Disease } \\
\text { Control and } \\
\text { Prevention }\end{array}$ & \\
\hline EPI710594 & $\mathrm{HA}$ & United States & 2016-Jan-16 & A/Wyoming/02/2016 & $\begin{array}{l}\text { Wyoming Public } \\
\text { Health Laboratory }\end{array}$ & $\begin{array}{c}\text { Centers for Disease } \\
\text { Control and } \\
\text { Prevention }\end{array}$ & \\
\hline EPI715789 & $\mathrm{HA}$ & Japan & 2016-Feb-16 & A/YOKOHAMA/87/2016 & $\begin{array}{c}\text { Yokohama City } \\
\text { Institute of Public } \\
\text { Health. }\end{array}$ & $\begin{array}{l}\text { National Institute of } \\
\text { Infectious Diseases } \\
\text { (NIID) }\end{array}$ & $\begin{array}{c}\text { Takashita,Emi; } \\
\text { Fujisaki,Seiichiro; } \\
\text { Shirakura,Masayuki; } \\
\text { Watanabe,Shinji; } \\
\text { Odagiri,Takato }\end{array}$ \\
\hline
\end{tabular}

